\title{
Role of Central Atom on the Pressure Response of 2D Perovskites Containing a Long Alkyl Chain (Decylammonium, DA): $\mathrm{DA}_{2} \mathrm{PbI}_{4}$ and $\mathrm{DA}_{2} \mathrm{GeI}_{4}$
}

Marta Morana, ${ }^{a,}{ }^{*}$ Rossella Chiara, ${ }^{a}$ Boby Joseph, ${ }^{b}$ Thomas B. Shiell, ${ }^{c}$ Timothy A. Strobel, ${ }^{c}$ Mauro Coduri, ${ }^{a}$ Gianluca Accorsi, ${ }^{d}$ Ausonio Tuissi, ${ }^{e}$ and Lorenzo Malavasi ${ }^{a,}{ }^{*}$

${ }^{a}$ Department of Chemistry and INSTM, Viale Taramelli 16, 27100, Pavia, Italy

${ }^{b}$ Earth and Planets Laboratory, Carnegie Institution for Science, Washington, DC 20015, USA

${ }^{c}$ Elettra-Sincrotrone Trieste S. C. p. A., S.S. 14 km 163.5 in Area Science Park, Basovizza, Trieste, Italy

${ }^{\mathrm{d} C N R}$ NANOTEC, Institute of Nanotechnology, c/o Campus Ecotekne, via Monteroni, Lecce, 73100, Italy

${ }^{\text {e}}$ CNR-Consiglio Nazionale delle Ricerche, Istituto di Chimica della Materia Condensata e di Tecnologie per l'Energia, Via Previati 1/e, 23900 Lecco, Italy

AUTHOR INFORMATION

Corresponding Authors

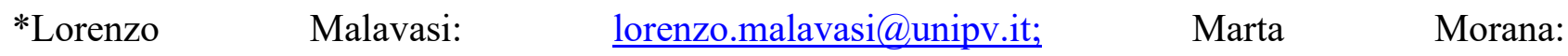

marta.morana01@universitadipavia.it 


\section{SUMMARY}

The application of an external pressure on Metal Halide Perovskite (MHPs) has become a fascinating way of tuning their optical properties, achieving also novel features. Here, the pressure response of 2D MHPs including a long alkyl chain made of ten carbon atoms, namely decylammonium (DA), has been investigated as a function of the central atom in $\mathrm{DA}_{2} \mathrm{PbI}_{4}$ and $\mathrm{DA}_{2} \mathrm{GeI}_{4}$. The two systems share a common trend in the phase stability, displaying a transition from an orthorhombic to a monoclinic phase around $2 \mathrm{GPa}$, followed by a phase separation in two monoclinic phases characterized by different $c$-axis. The optical properties show rather different behavior due to the presence of $\mathrm{Pb}$ or Ge. $\mathrm{DA}_{2} \mathrm{PbI}_{4}$ shows a progressive red shift of the band gap from $2.28 \mathrm{eV}$ at ambient conditions, to $1.64 \mathrm{eV}$ at $11.5 \mathrm{GPa}$, with a narrow PL emission composed by two components, with the second one appearing in concomitance with the phase separation and significantly shifted to lower energy. On the other hand, $\mathrm{DA}_{2} \mathrm{GeI}_{4}$, changes from a non-PL system at ambient pressure, to a clear broadband emitter centered around $730 \mathrm{~nm}$ (FWHM $170 \mathrm{~nm}$ ), with a large stoke shift, and an intensity maximum at about $3.7 \mathrm{GPa}$. This work sheds light on the structural stability of 2D perovskites characterized by extended alkyl chains, to date limited to four carbon atoms, and shows the pressure-induced emergence of broad emission in a novel lead-free perovskite, $\mathrm{DA}_{2} \mathrm{GeI}_{4}$. The evidence of wide emission by a moderate pressure in a germanium-based 2D MHP represents a novel result which may open the design, by chemical pressure, of efficient wide or even white lead-free emitters.

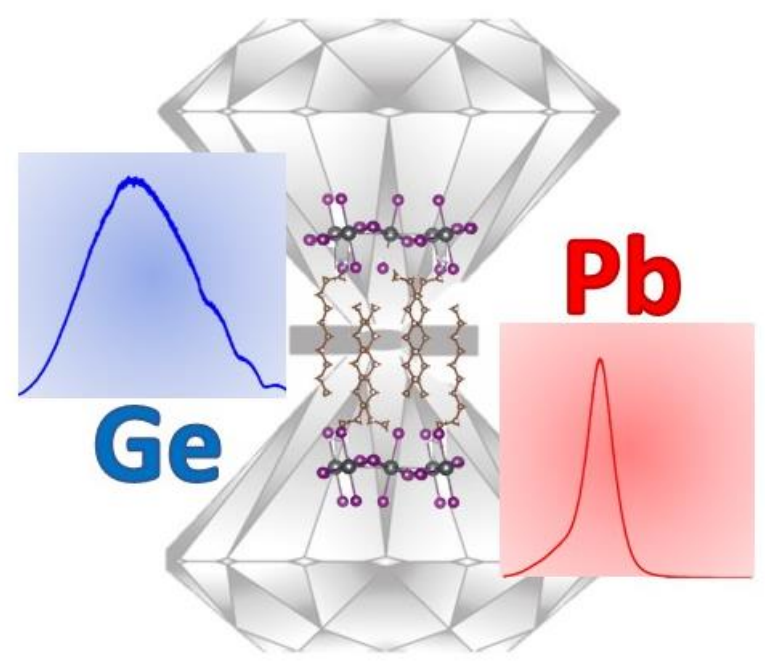




\section{Introduction}

The exploration of the effect of external pressure application on metal halide perovskites (MHPs) is a topic of continuous interest due to the relevant and in some cases impressive modulation of the optical and electronic properties that can be induced on the MHP soft lattice at relatively low-pressure regimes. ${ }^{1-5}$ The rich set of experimental and computation data as a function of pressure collected on $3 \mathrm{D}$ perovskites of general formula $\mathrm{ABX}_{3} \quad(\mathrm{~A}=$ methylammonium, formamidinium, cesium, etc...; $\mathrm{B}=\mathrm{Pb}, \mathrm{Sn}$, and $\mathrm{Ge} ; \mathrm{X}=\mathrm{Cl}, \mathrm{Br}$, and I) allowed to highlight and define several common trends in the pressure-response of, for example, band gap and carrier lifetime, providing a solid basis to anticipate and predict the phase stability and electronic properties changes in these phases. ${ }^{1-9}$

On the other hand, the study of pressure effects on the electronic and optical properties of so-called low-dimensional perovskites (LDP) and perovskite-derivatives is very limited notwithstanding the current huge interest related to the vast possibility provided by the variation of the structural dimensions (2D, 1D, and 0D) and octahedral connectivity (corner-sharing, edgesharing, and face-sharing) in this class of materials. ${ }^{10-13}$ Among the different types of LDPs, the 2D or layered systems of the Ruddlesden-Popper (RP) type are a hot topic of research due their high application potential in various fields ranging from photovoltaics, optoelectronic to photodetection..$^{10,11,14-16}$ The available data about the pressure response of LDPs has been recently reviewed by Zhang and co-workers. ${ }^{17}$ Different from 3D perovskites, where the variation of the bond lengths and bond angles of the inorganic framework upon pressure application plays the key role in the modulation of properties, the presence of a bulky organic component, layered among the inorganic layers in 2D perovskites, provides a further degree of structural complexity and may provide novel routes for the tuning of functional properties. The number of 2D MHPs prepared and investigated to date, particularly lead-based materials, is impressive. These studies, which include 
the rational design of the organic cation and halide, have shed light on the structure-property correlation in these systems providing a solid route to design tailored materials. ${ }^{10}$

On the other hand, the number of high-pressure studies of 2D MHPs of the RP series, with $n=1$, i.e. compounds of general formula $\mathrm{A}_{2} \mathrm{BX}_{4}$, is very limited and only focused on a few types of organic spacers, even though these specific LDP are among the structural series of major interest for applications. ${ }^{17}(\mathrm{BA})_{2} \mathrm{PbI}_{4}$ (BA=butylammonium) has been object of intense High Pressure (HP) studies by various authors since $2004 .{ }^{18-20}$ A thorough investigation by Yuan and co-workers up to $40 \mathrm{GPa}$ indicates the presence of three phase transitions at $0.22,2.2$ and $13.1 \mathrm{GPa}$, with the coexistence of phase II and III in the range 2-7 GPa, however it should stressed that the sample shows a clear on-set of amorphization already from about $4 \mathrm{GPa}^{20}$ The band gap of $(\mathrm{BA})_{2} \mathrm{PbI}_{4}$, determined from absorbance measurements, first presents a blue-shift at about $0.22 \mathrm{GPa}$ from 2.28 to $2.37 \mathrm{eV}$, followed by a progressive band gap narrowing up to $0.95 \mathrm{eV}$ at around $35 \mathrm{GPa}^{20}$ The trend of photoluminescence (PL) data confirms the behavior of absorbance measurements with a peak in the PL intensity around $2 \mathrm{GPa}$, which eventually vanishes around 6-7 GPa. Both PL and absorbance data point towards the presence of a wide two-phase region below $10 \mathrm{GPa}$ as evidenced by HP xray diffraction (XRD) data. An analogous 2D perovskite with a longer alkyl chain (octylammonium) has been investigated up to $25 \mathrm{GPa}$ in $2001 .^{21}$ The authors reported the trend of PL and absorbance only, without any structural evidence as a function of $P$. A progressive reduction of the band gap from about 2.4 to $1.9 \mathrm{eV}$ is observed in the $P$ range from ambient pressure to about $12 \mathrm{GPa}^{21}$ From the reported data it is possible to observe the presence of multiple absorption edges as well as PL peaks (up to three) from about $2 \mathrm{GPa}$ to about $8 \mathrm{GPa}$ which are interpreted by the authors as originating from bound-excition states. Other phenomena such as the disappearance of the excitonic state are discussed in terms of a change in the band structure from direct to indirect. Alternatively, as can be indirectly inferred from the reported plots of the optical properties, the observed trends could be due to the presence of a system composed by more than one structural 
phase as occurs in the $(\mathrm{BA})_{2} \mathrm{PbI}_{4}$. It is interesting to note that in these $\left(\mathrm{C}_{n} \mathrm{H}_{2 n+1} \mathrm{NH}_{3}\right)_{2} \mathrm{PbI}_{4}$ perovskites for $n=4$ and 8 the trend of the band gap is different with respect to lead-based 3D perovskites where, after a red-shift at relatively low pressures, a progressive blue-shift occurs, usually accompanied by structural amorphization. ${ }^{1}$ The presence of the organic spacer, while not directly influencing the electronic structure, can play a templating role by affecting the inorganic sublattice, which indirectly modulates the optoelectronic properties in metal halide perovskites. In this respect it is of key importance to try to understand in a systematic way the role of the organic spacer on the properties of 2D MHPs. For example, the structural and optical studies carried out on the $\left(\mathrm{C}_{n} \mathrm{H}_{2 n+1} \mathrm{NH}_{3}\right)_{2} \mathrm{PbI}_{4}$ perovskites as a function of the number of carbon atoms $(n)$ of the alkylammonium cation, helped researchers to rationalize the role of chain length on the properties of $2 \mathrm{D}$ perovskites such as electronic structure, exciton binding energies, orientation and melting of the organic chain, phase transitions, etc....22-25

Triggered by the actual interest on LDPs and by the limited evidence of a relevant pressureresponse in these systems, we conducted a $\mathrm{HP}$ study of the $\left(\mathrm{C}_{10} \mathrm{H}_{21} \mathrm{NH}_{3}\right)_{2} \mathrm{PbI}_{4} 2 \mathrm{D}$ perovskite, i.e. containing the decylammonium (DA) cation, by means of XRD, PL and absorbance measurements as a function of pressure. Moreover, since the metal central atom plays a crucial role on the electronic structure of MHPs, we were interested in comparing the HP effects for similar systems but containing a different metal, namely germanium. We recently started to investigate the structure-property correlation in Ge-based 2D perovskites with the aim of understanding how the replacement of this ion for lead affects the structural, electronic, and optical properties, also considering the significant environmental problem raised by lead use and the necessity of finding possible lead-free alternatives. ${ }^{26,27}$ To the best of our knowledge, the present paper not only provides the first high-pressure experimental work on $\mathrm{DA}_{2} \mathrm{PbI}_{4}$ and $\mathrm{DA}_{2} \mathrm{GeI}_{4}$, the latter being a novel perovskite, but also represents the only available work of the pressure response of a Ge-based 2D MHPs. 


\section{Results and Discussion}

High Pressure XRD and Optical Characterization of $\mathrm{DA}_{2} \mathrm{PbI}_{4}$

$\mathrm{DA}_{2} \mathrm{PbI}_{4}$ has been prepared by wet-chemistry route, as described in the Experimental Section, and the ambient temperature and pressure XRD pattern can be refined in the orthorhombic Pbca space group as reported previosuly (see Figure S1 of Supporting Information, SI). ${ }^{25}$ Highpressure XRD data have been collected at the XPress beamline, Elettra synchrotron, from room pressure to $9.4 \mathrm{GPa}$ by using silicone oil as pressure transmitting medium (PTM) at the wavelength of $0.495 \AA$ (further details are provided in the SI). An overview of the diffraction patterns collected as a function of the applied pressure is reported in Figure 1, also including the pattern after pressure release ("0 GPa decomp" at the top of Figure 1).
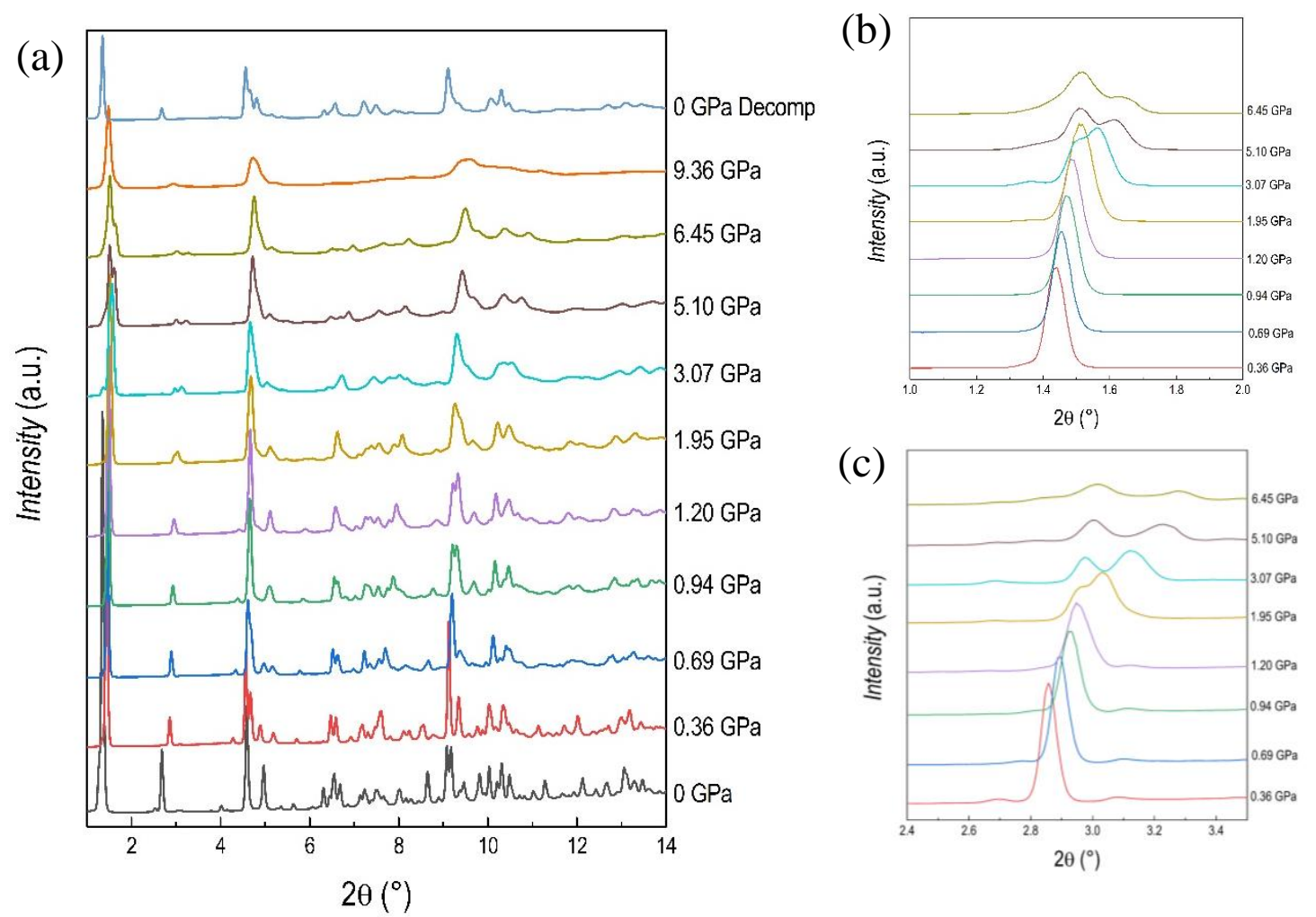

Figure 1 -(a) XRD patterns $(\lambda=0.495 \AA$ ) as a function of pressure (reported in GPa on the right) for $\mathrm{DA}_{2} \mathrm{PbI}_{4}$; (b) and (c) highlights selected intervals of the pattern to better shown the evolution of the (001) and (002) reflections as a function of pressure. 
Already at the first pressure investigated, namely $0.36 \mathrm{GPa}$, the crystal structure can no longer be indexed to the ambient conditions orthorhombic Pbca cell, and a monoclinic cell in the space group $P 2{ }_{1} / a$ has been found to properly describe the crystal structure. This symmetry is very close to the one obtained from single crystal data at low temperature $(243 \mathrm{~K}) .{ }^{25}$ A similar transition has been reported also for the $(\mathrm{BA})_{2} \mathrm{PbI}_{4}$ at $\approx 2 \mathrm{GPa}$, again in analogy with the phase transition sequence as a function of temperature..$^{20,23}$ The refinement of the data at $0.36 \mathrm{GPa}$ was then performed in the space group $P 2{ }_{1} / a$, obtained from single crystal data (see Figure $S 2$ ). ${ }^{25}$ According to the structural study by Lemmerer and Billing, this $P b c a \rightarrow P 2_{1} / a$ transformation is mostly due to changes in the inorganic layers that assume an eclipsed arrangement of adjacent layers, while the unit cell axis halves perpendicular to the layers. ${ }^{25}$ The $\mathrm{DA}_{2} \mathrm{PbI}_{4}$ sample retains the same symmetry up to $1.70 \mathrm{GPa}$, when the peaks at approximately $1.5^{\circ}$ and $3^{\circ}$, corresponding to the single (001) and (002) reflections in the $P 2_{1} / a$ symmetry, start to show a splitting that increases upon compression (Figures $1 \mathrm{~b}$ and 1c). This phenomenon clearly indicates the presence of two distinct phases in the sample. As a consequence, the patterns were now refined with two phases with space group $P 2{ }_{1} / a$ characterized by the $c$-axes differing approximately $2 \AA$ each other. Notably, the intensity of the (001) reflection initially decreases till $3.07 \mathrm{GPa}$, then it increases again upon further compression indicating a variation of the relative phase amount with pressure. In this respect, considering the past literature data and the results of the present manuscript, it seems that a common trend of $\left(\mathrm{C}_{n} \mathrm{H}_{2 n+1} \mathrm{NH}_{3}\right)_{2} \mathrm{PbI}_{4}$ perovskites is the evolution, upon the application of pressure, to multiple-phase systems. $^{21,25}$ At $6.45 \mathrm{GPa}$ the broadening of the peaks increases, and this effect appears more significant at $9.36 \mathrm{GPa}$, suggesting the onset of partial amorphization. The evolution of the cell parameters and lattice volume is summarized in Fig $2 \mathrm{a}$ and $2 \mathrm{~b}$. 
(a)

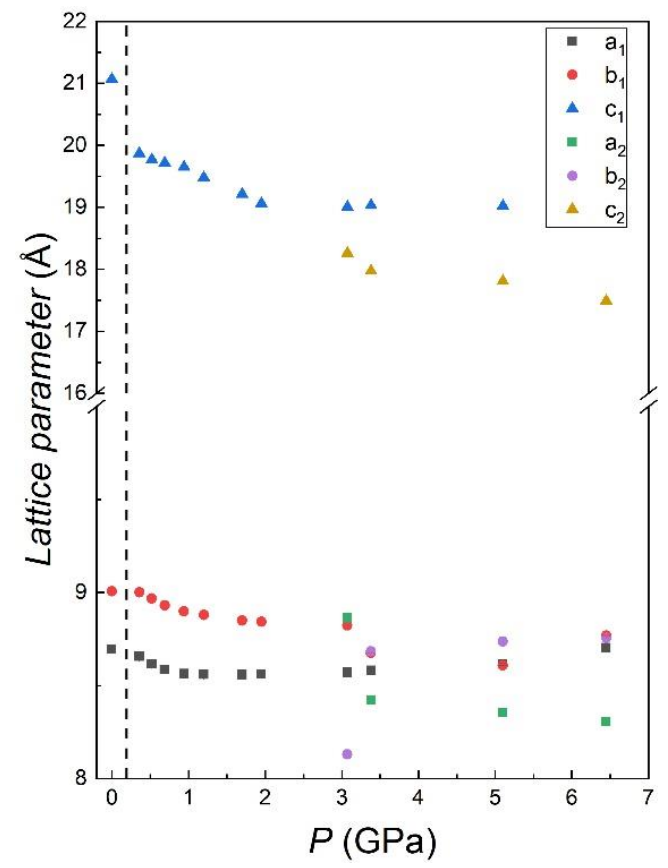

(b)

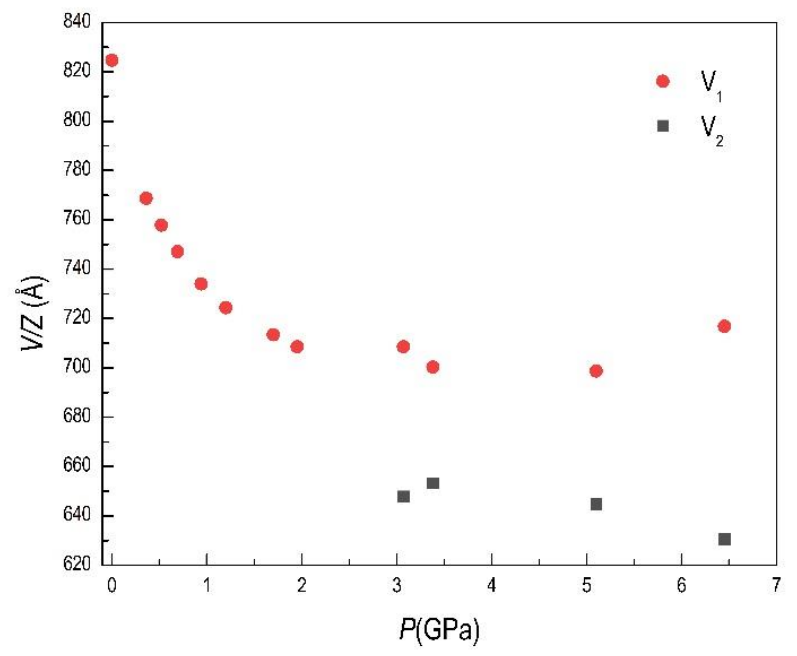

Figure 2 -Pressure dependence of the cell parameters $a, b, c$ (a) and of the cell volume (b) for $\mathrm{DA}_{2} \mathrm{PbI}_{4}$. The $c$ axis of the orthorhombic cell was halved. The dashed line marks the transition from the orthorhombic to the monoclinic cell. Subscripts 1 and 2 refer to the two monoclinic phases.

After pressure release, the pattern can be indexed again with the orthorhombic cell, suggesting at least a certain degree of reversibility of the transition (Figure S3). However, the longrange structural integrity of the sample has been reduced after pressure application, but the position of the most intense peaks in the pattern $(00 l)$ can be still found at the same positions as the starting sample (Figure S3).

The optical properties as a function of pressure have been investigated by means of absorption and PL spectroscopies and the data are reported in Figures $3 \mathrm{a}$ and $4 \mathrm{a}$, respectively. 
(a)

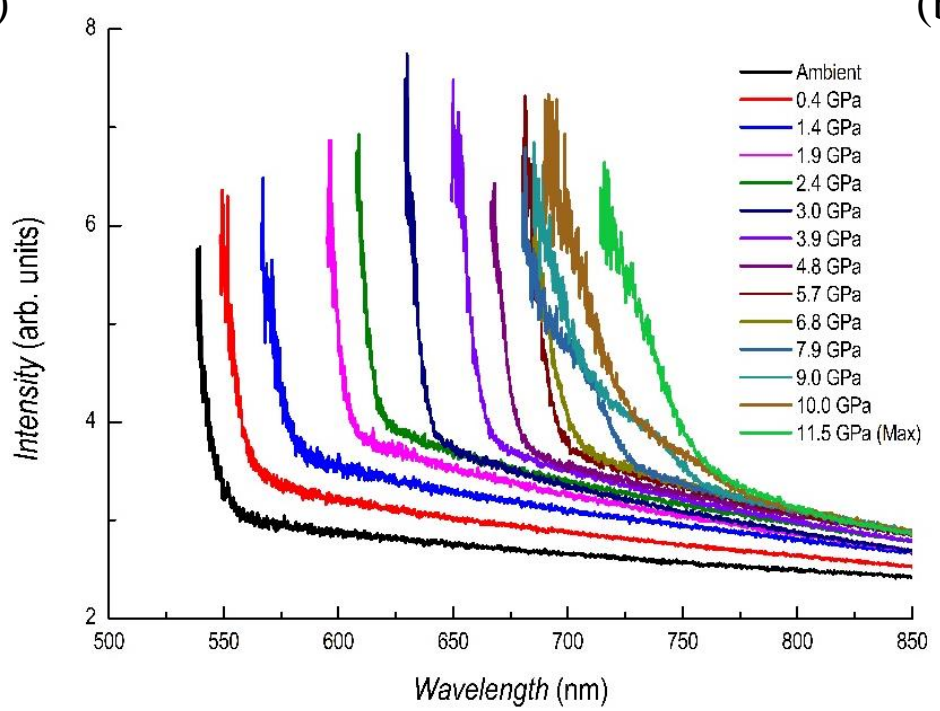

(b)

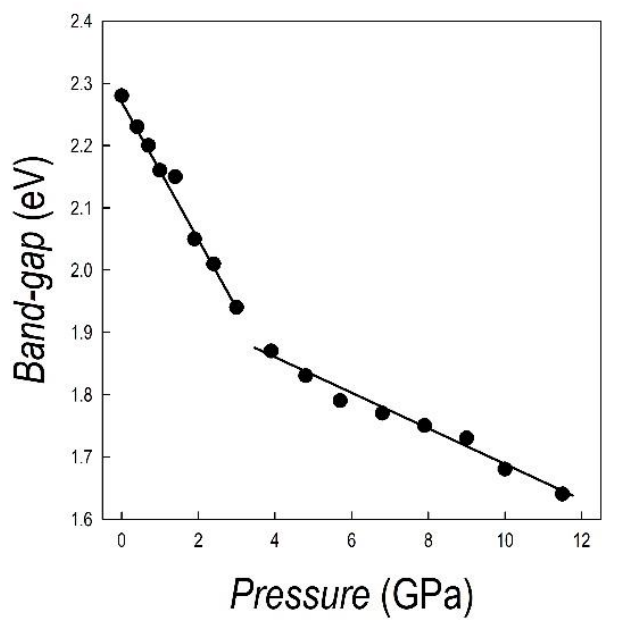

Figure 3 - (a) absorption spectra as a function of pressure for $\mathrm{DA}_{2} \mathrm{PbI}_{4}$; (b) trend of the band gap extracted from the spectra as a function of pressure.

The absorption data show a progressive and continuous red shift by increasing pressure. In general, the spectra show sharp edges even in the two-phase region determined by XRD, differently from the PL results (see below). A significant broadening of the spectra, with the appearance of a shoulder, is evident only in the spectra at 7.9 and $9 \mathrm{GPa}$. The trend of the band gap energy determined from the Tauc plot of the spectra of Figure 3a are reported in Figure $3 \mathrm{~b}$. The band gap energy progressively decreases from the ambient pressure value of $2.28 \mathrm{eV}$, in agreement with previous literature data, down to $1.64 \mathrm{eV}$ at $11.5 \mathrm{GPa}{ }^{22}$ No sign of variation is found in the region corresponding to the $\mathrm{Pbca} \rightarrow P 2_{1} / a$ phase transition $(0-0.36 \mathrm{GPa})$, while a different trend in the band gap variation is observed (see continuous lines in Figure 3b) around the region where a twophase system is found according to diffraction.

The PL spectra as a function of pressure are shown in Figure 4a. A single PL peak is found up to $1.4 \mathrm{GPa}$ (blue curve in Figure 4a) while from 1.9 GPa two distinct peaks are clearly visible in the spectra, with the second one shifted at higher wavelengths. This phenomenon is most probably related to the presence, starting from $1.9 \mathrm{GPa}$, of two distinct monoclinic phases in the sample, as shown by XRD analysis, with PL being more sensitive than absorbance in detecting this phase 
separation effect. The evolution of the peak position, expressed in $\mathrm{eV}$, as a function of pressure is plotted in Figure 4b.

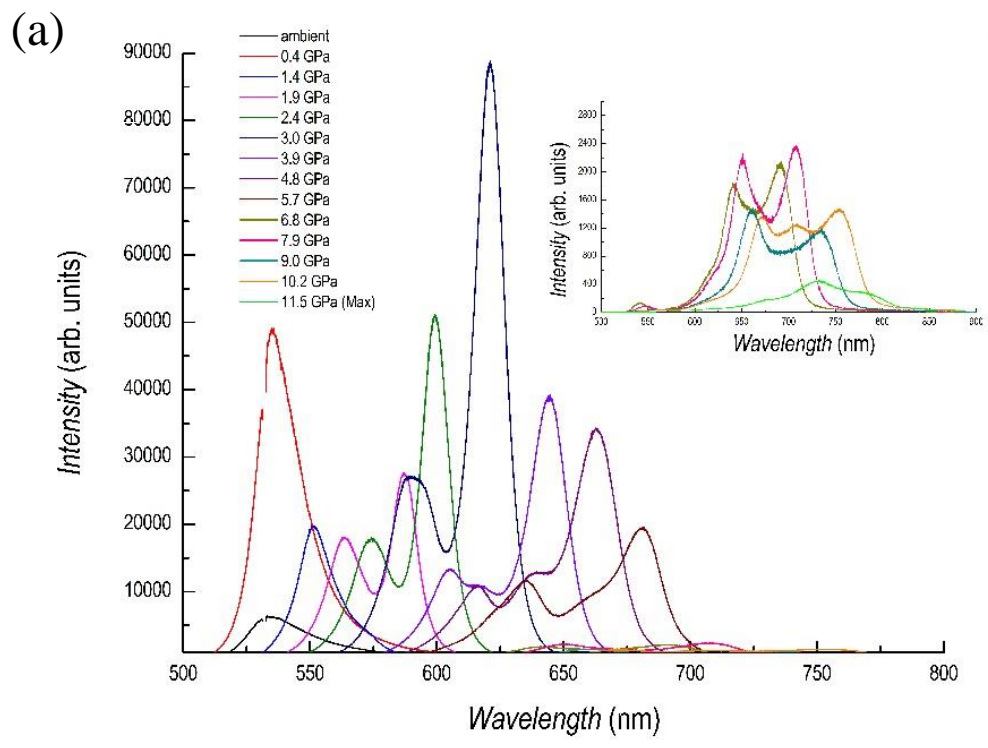

(b)

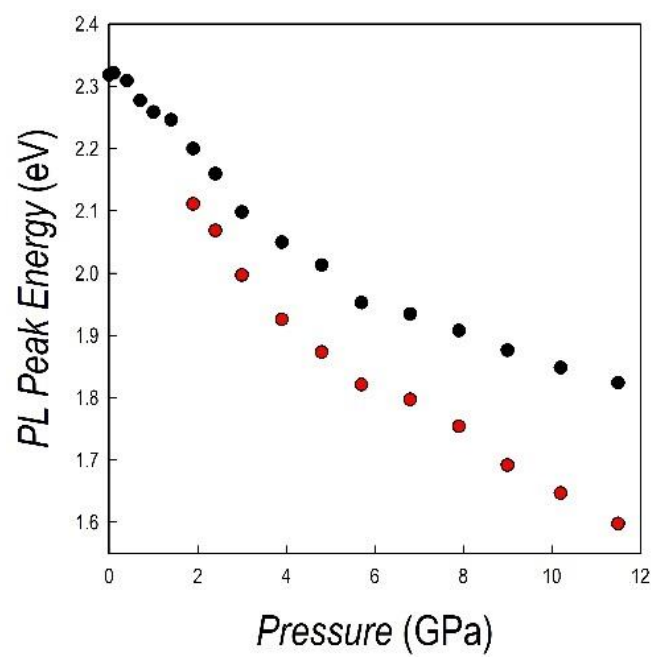

Figure 4 - (a) PL spectra as a function of pressure for $\mathrm{DA}_{2} \mathrm{PbI}_{4}$; (b) trend of the peak position of the PL data as a function of pressure.

The intensity of the first peak (lower wavelength) increases passing from ambient pressure to $0.4 \mathrm{GPa}\left(\mathrm{Pbca} \rightarrow P 2_{1} / a\right.$ transition) and then gradually reduces its intensity. On the other hand, the low-energy peak intensity progressively increases up to $3 \mathrm{GPa}$ and then decreases up to $5.7 \mathrm{GPa}$. The PL intensity of both peaks suddenly drops at $6.8 \mathrm{GPa}$ even though the peaks continue to shift at lower energy (see the inset of Figure 4b). The trend of the first PL peak (black dots in Figure 4b) as a function of pressure is very similar to the trend of the band gap extracted from the absorbance data, with a slope deviation around $2 \mathrm{GPa}$. Possibly, the second peak could be ascribed to the second monoclinic phase with a more compressed $c$-axes characterized by a low energy band gap (red dots in Figure 3b). 
The trend of the absorbance and PL results may be interpreted considering the progressive $\mathrm{Pb}-\mathrm{I}$ bond length shortening induced by pressure which gradually narrows the band gap, as commonly found in 3D perovskites. ${ }^{1}$ The presence of two distinct PL peaks is related to the presence of two different phases in the sample from about $2 \mathrm{GPa}$. As can be seen, the two PL peak maxima are well separated and the one at higher wavelength has a more pronounced red-shift with respect to the peak at lower wavelength. As we demonstrated with in situ XRD data, the two monoclinic phases have a difference in the $c$-axis of about $2 \AA$. It is reasonable to ascribe the lowenergy PL peak to the more compressed phase, also considering the trend of the $c$-axes of both phases shown in Figure 2a, which in some way reconciles with the behavior of the PL maxima of the two peaks. Some of the results reported here for $\mathrm{DA}_{2} \mathrm{PbI}_{4}$ have been observed also in $\mathrm{BA}_{2} \mathrm{PbI}_{4}$ (BA=butylammonium), namely the coexistence of two phases, the trend of the peak positions and intensity. A marked difference between the two samples is related to the absence of any blue-shift at low-pressure. The presence of a blue-shift is common in 3D perovskites and is related to the increase deviation from $180^{\circ}$ of $\mathrm{Pb}-\mathrm{I}-\mathrm{Pb}$ bond angle, widening the band gap. ${ }^{1}$ For a short alkyl chain, namely four carbon atoms, such an effect is still present while, in $\mathrm{DA}_{2} \mathrm{PbI}_{4}$, having a longer chain, only a progressive red-shift has been observed. Moreover, it is interesting to note that such red-shift is present passing from the orthorhombic to the monoclinic phase and is continuous in such a crystal symmetry. In general, the more distorted octahedra of the monoclinic phase leads to an increase of the band gap, which is not the present case. It is highly probable that the origin of this effect is related to the variation of the organic spacer conformation which progressively becomes more distorted while leading to less tilted and more compressed $\mathrm{PbI}_{6}$ octahedra and therefore band gap narrowing. This hypothesis has been formulated for the $\mathrm{BA}_{2} \mathrm{PbI}_{4}$ perovskite where, by increasing pressure, even in a more distorted phase, the octahedra result to be uncorrugated but, due to the low scattering contribution of the organic spacer, it is hard to gain solid insight into this phenomeon. ${ }^{20}$ A conclusion which may be drawn from the reported data is that a longer organic chain, in lead-based 2D perovskites, allows to reduce the tilting effects induced by pressure 
application on the inorganic framework (intralayer compression), making the layer-to-layer compression dominant and as the source of the observed continuous red-shift. To corroborate this observation it is possible to mention that, by increasing the number of inorganic layers in the $(\mathrm{BA})_{2}(\mathrm{MA})_{\mathrm{n}-1} \mathrm{~Pb}_{\mathrm{n}} \mathrm{I}_{3 \mathrm{n}+1}$ system, i.e. making the 2D progressively "more $3 \mathrm{D}$ ", the pressure range of the red-shift and the value at which the band gap blue-shift occurs progressively lower. ${ }^{28}$

High Pressure XRD and Optical Characterization of $\mathrm{DA}_{2} \mathrm{GeI}_{4}$

An analogous experimental approach has been used to investigate the HP behavior of $\mathrm{DA}_{2} \mathrm{GeI}_{4}$, which presents as an orange powder. $\mathrm{DA}_{2} \mathrm{GeI}_{4}$ is a novel material and its crystal structure has never been determined before. In addition, as mentioned above, this is the first study of the pressure response of any Ge-based 2D perovskite. Indexing and refinement of powder diffraction data at ambient pressure suggest that the compound crystallize in the orthorhombic system $(P b c a)$, as the lead analogue $\mathrm{DA}_{2} \mathrm{PbI}_{4}{ }^{25}$ The refined pattern at ambient pressure is reported in Figure S4.

HP XRD data have been collected up to $11.1 \mathrm{GPa}$ every $\sim 0.5 \mathrm{GPa}$ and some selected patterns are reported in Figure 5a. 
(a)

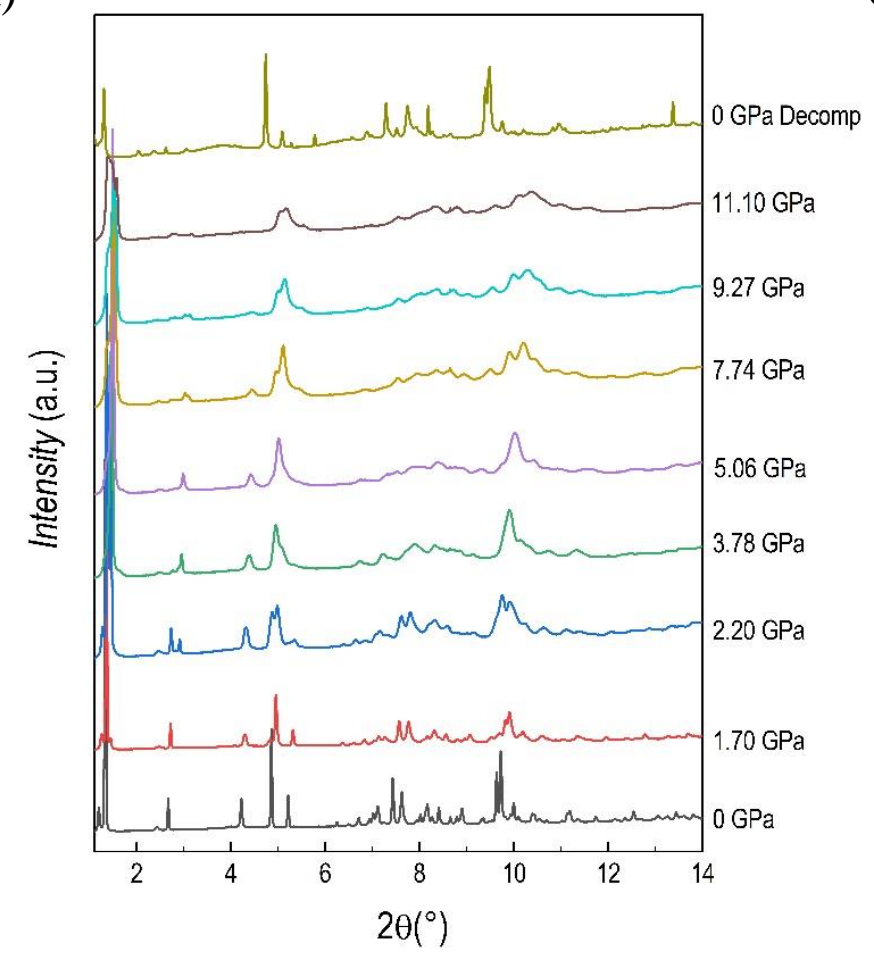

(b)

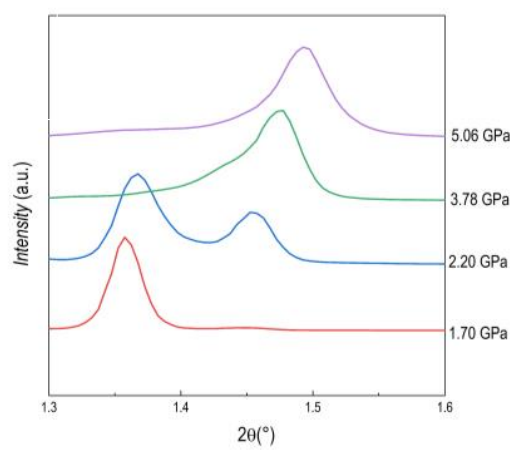

(c)

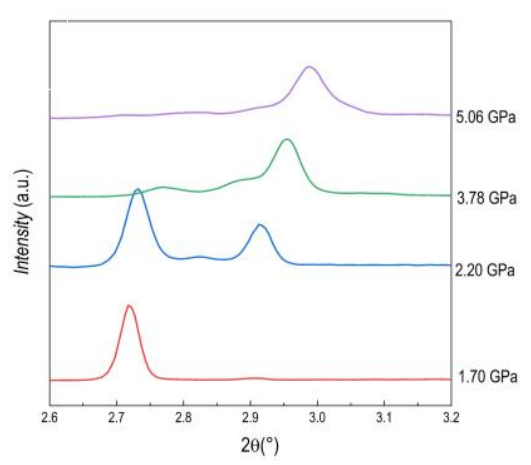

Figure 5 -(a) XRD patterns $(\lambda=0.495 \AA)$ as a function of pressure (reported in GPa on the right) for $\mathrm{DA}_{2} \mathrm{GeI}_{4}$; (b) and (c) highlights selected intervals of the pattern to better shown the evolution of the (001) and (002) reflections as a function of pressure.

The first high pressure pattern at $0.56 \mathrm{GPa}$ shows a monoclinic symmetry in analogy with $\mathrm{DA}_{2} \mathrm{PbI}_{4}$ (see refined pattern as Figure S5). The sample persists as a single phase up to $2.20 \mathrm{GPa}$, when a splitting of the peaks at approximately $1.4^{\circ}$ and $2.8^{\circ}$ is clearly detectable (Fig $5 \mathrm{a}$ and $5 \mathrm{~b}$ ). Beyond this pressure, the patterns were refined with two phases with space group $P 2{ }_{1} / a$, where the $c$ axes differ by approximately $1 \AA$. In particular, the intensity of the (001) reflection of the first phase decreases, while the (001) reflection of the second phase increases (Fig 5b) as a function of pressure. Already around $3.8 \mathrm{GPa}$ the phase with longer $c$-axes represents a significantly minority phase. A similar behavior occurs for the (002) reflections around $2.8^{\circ}$ (Fig 5c). The two phases clearly coexist up to $5.06 \mathrm{GPa}$ when the broadening of the peaks increases. The trend of lattice parameters and lattice volume as a function of pressure are reported in Figures $6 \mathrm{a}$ and $6 \mathrm{~b}$, respectively. 
(a)

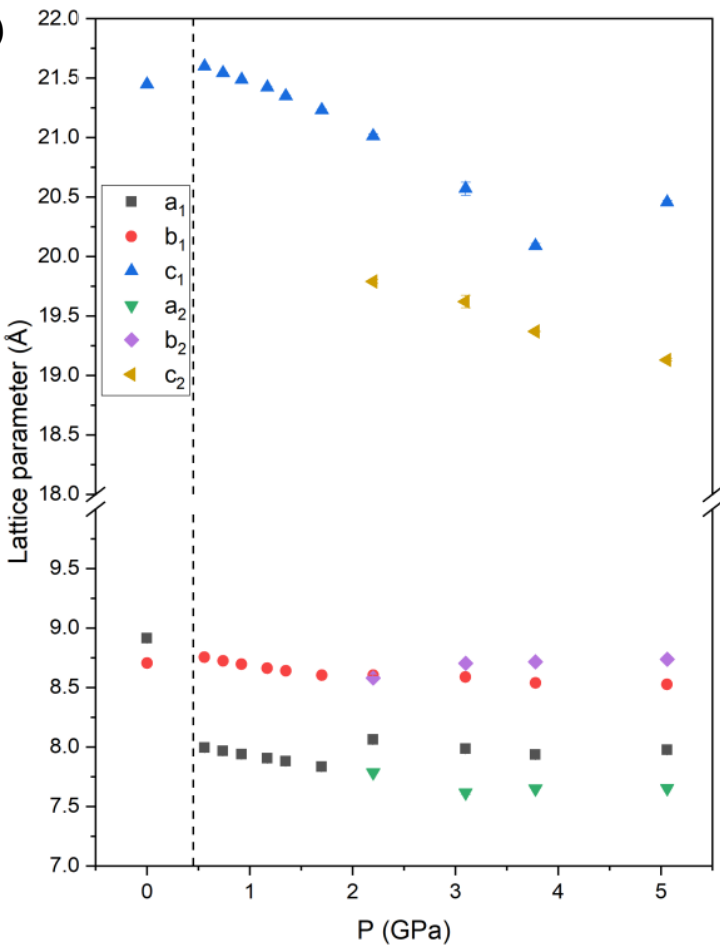

(b)

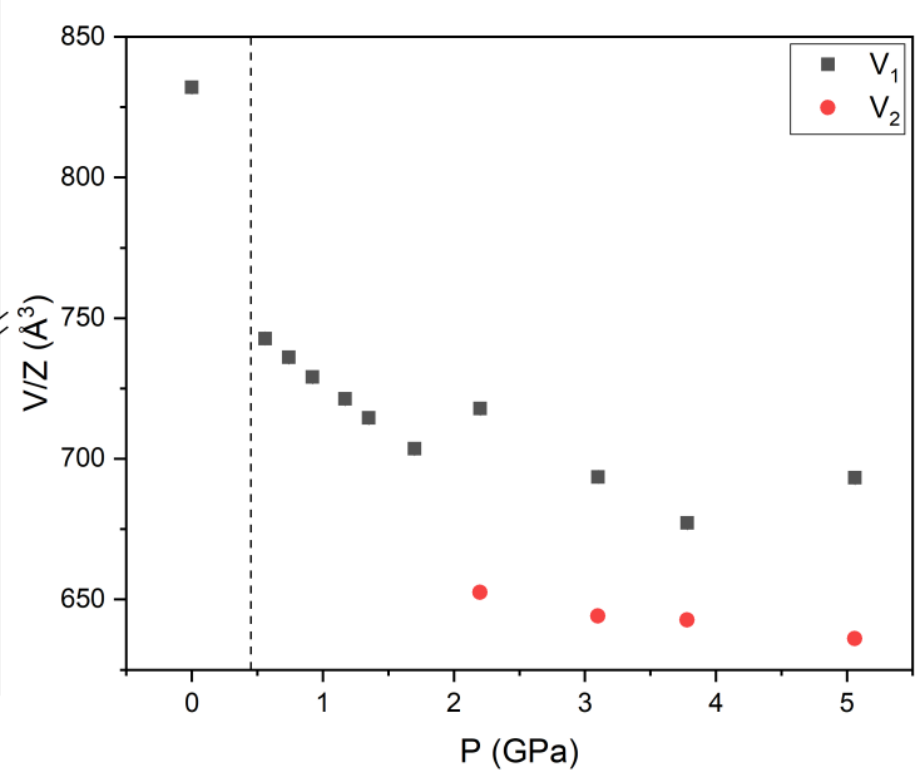

Figure 6 -Pressure dependence of the cell parameters $a, b, c$ (a) and of the cell volume (b) for $\mathrm{DA}_{2} \mathrm{GeI}_{4}$. The $c$ axis of the orthorhombic cell was halved. The dashed line marks the transition from the orthorhombic to the monoclinic cell.

As mentioned above, $\mathrm{DA}_{2} \mathrm{GeI}_{4}$ is a novel compound and to get some insight into the structural behavior observed under pressure we collected variable-temperature laboratory XRD patterns. The most interesting result has been observed by heating the sample from room temperature (RT). Data are reported in Figure S8. At $50^{\circ} \mathrm{C}$ we could observe a phase separation of the sample, analogous to what has been observed by applying pressure. In general, such an effect corresponds to a disordering or even melting of the organic spacer. ${ }^{23-25}$ Further heating to $80^{\circ} \mathrm{C}$ leads to the restoration of a single phase (at least with the detection limits of our measurements). The common coexistence of two structural phases by the disordering/melting of the organic spacer by applying pressure and by increasing temperature is an interesting result which merits a further detailed investigation. As a matter of fact, it is known that in $\left(\mathrm{C}_{n} \mathrm{H}_{2 n+1} \mathrm{NH}_{3}\right)_{2} \mathrm{PbI}_{4}$ perovskites there is 
a complex and rich phase transition behavior due not only to the inorganic framework but also to the ordering of the organic spacer. ${ }^{23-25}$

The optical properties as a function of pressure have been studied by absorption (Figure 7a) and PL (Figure 8b) spectroscopy.

(a)

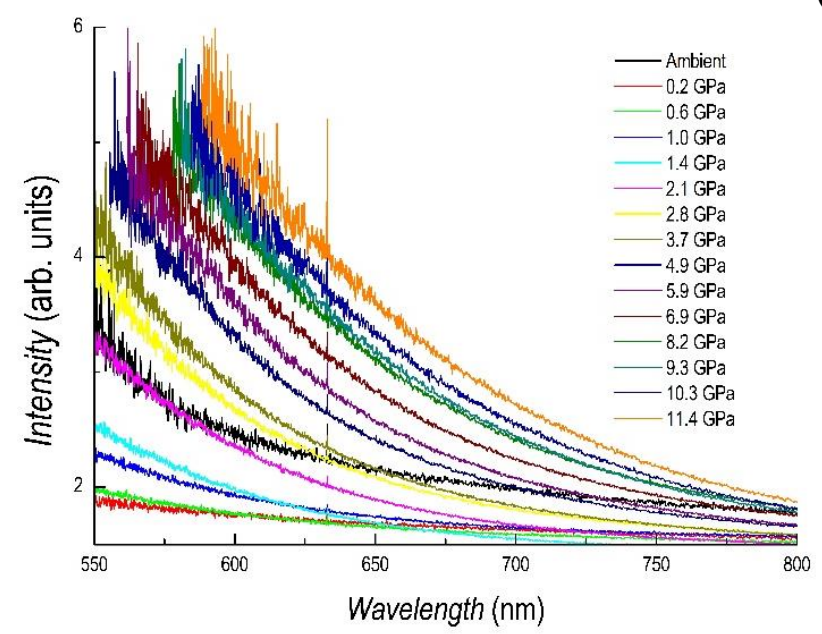

(b)

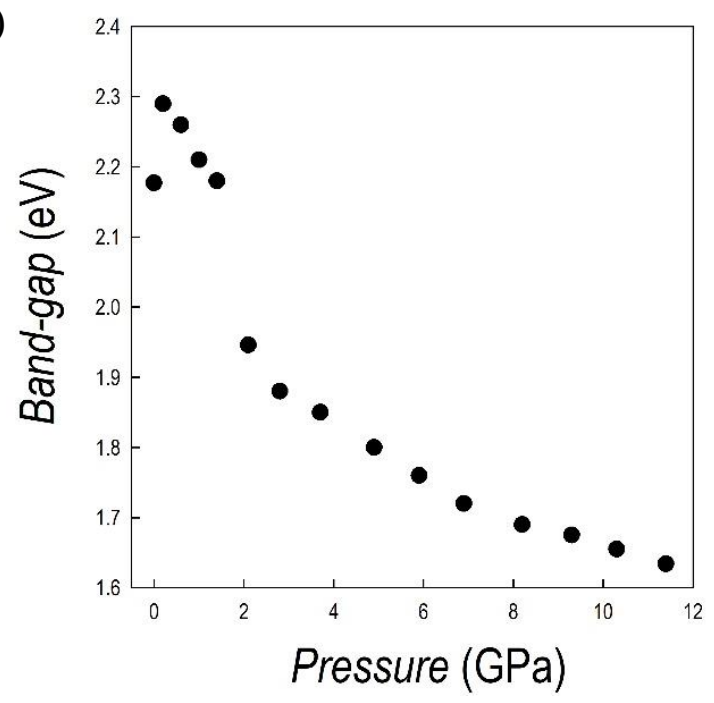

Figure 7 - (a) absorption spectra as a function of pressure for $\mathrm{DA}_{2} \mathrm{GeI}_{4}$. The peak at $633 \mathrm{~nm}$ is from the HeNe calibration laser; (b) trend of the band gap extracted from the spectra as a function of pressure.

The spectra as a function of pressure for $\mathrm{DA}_{2} \mathrm{GeI}_{4}$ show a sudden blue-shift after the application of a moderate pressure $(0.2 \mathrm{GPa})$ which may correspond to the set-up of the orthorhombic to monoclinic transition, differently from $\mathrm{DA}_{2} \mathrm{PbI}_{4}$. Further pressure application gradually red-shift the absorbance and, at $2.1 \mathrm{GPa}$, the spectra is nearly superimposable onto that collected at ambient pressure (see Figure 3a). The red-shift is then continuous until the maximum pressure investigated $(11.4 \mathrm{GPa})$. With respect to $\mathrm{DA}_{2} \mathrm{PbI}_{4}$, the absorption edges are less defined and this is a feature quite common in lead-free perovskites with a possible origin in their reduced defect-tolerance. ${ }^{29}$ The values of the band gap extracted from the Tauc plots (except for some points at low pressure where, given the broad nature of the transitions, we used the maximum absorbance approach) show a change of slope and a sudden decrease in correspondence of about $2 \mathrm{GPa}$, i.e. the 
pressure where the patterns were refined with two phases with space group $P 2{ }_{1} / a$ and the $c$ axes that differ by approximately $1 \AA$ (see above the XRD section). As we pointed out earlier, the phase with shorter $c$ axis is dominant around $3 \mathrm{GPa}$, and this reconciles with the absence of multiple absorption edge in the spectra of Figure 7a.

The trend of $\mathrm{PL}$ data for $\mathrm{DA}_{2} \mathrm{GeI}_{4}$ is reported in Figure 8a. At ambient pressure the sample has no detectable PL at room temperature while, from $2.1 \mathrm{GPa}$, a very broad peak, centered around $740 \mathrm{~nm}(1.67 \mathrm{eV})$ and with a Stokes shift $>70 \mathrm{~nm}$, emerges and reaches it maximum intensity around 3.7 GPa. The Full Width at Half Maximum (FWHM) of the peak is $~ 170 \mathrm{~nm}$ and both peak position and FWHM have a very modest dependance with pressure. Such behavior is fully reversible during decompression (see Figure S9) and, for comparison, the peak position at $3.7 \mathrm{GPa}$ is $737 \mathrm{~nm}$ (compression), and $732 \mathrm{~nm}$ at $4.4 \mathrm{GPa}$ (decompression), and the FWHM are 156 and 152 $\mathrm{nm}$, respectively.

(a)

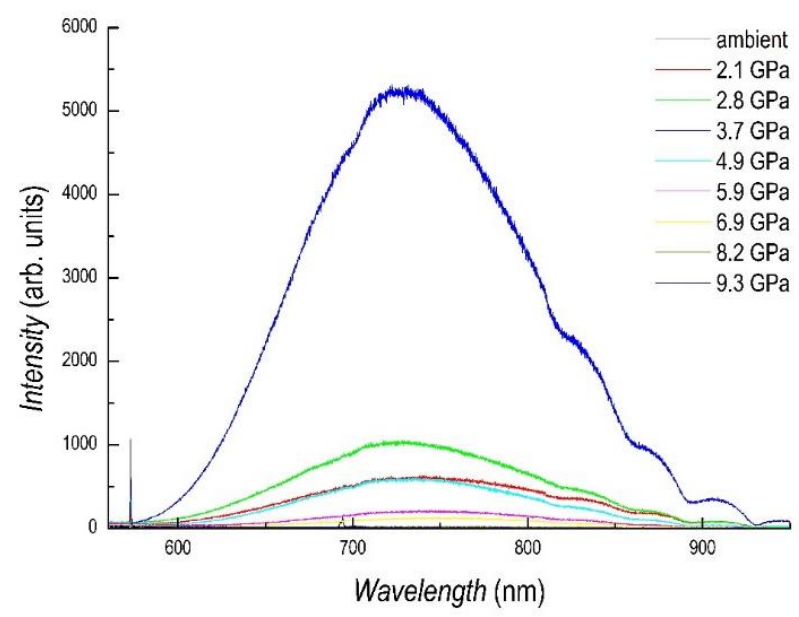

(b)

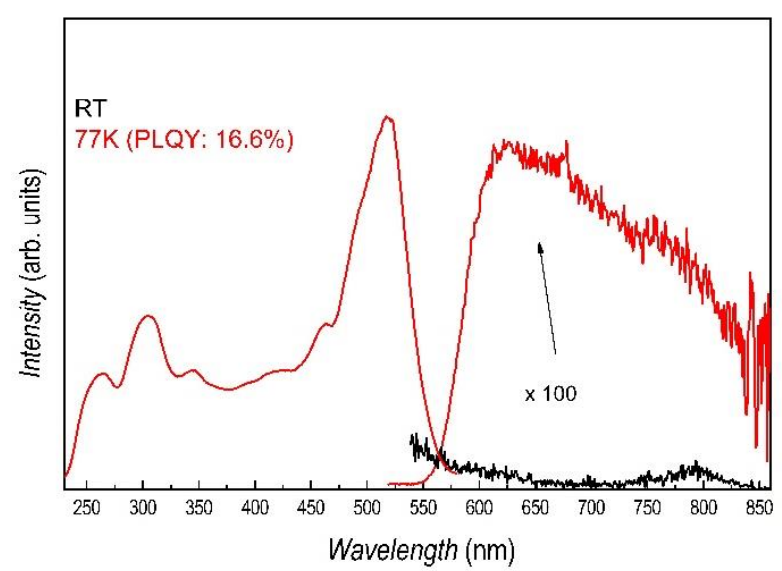

Figure 8 - (a) PL data under compression for $\mathrm{DA}_{2} \mathrm{GeI}_{4}$. Oscillations above $\sim 800 \mathrm{~nm}$ are caused by detector etaloning; (b) PL data at room temperature (black line) and $77 \mathrm{~K}$ (red line) for $\mathrm{DA}_{2} \mathrm{GeI}_{4}$.

The evidence of a broadband emission by $\mathrm{DA}_{2} \mathrm{GeI}_{4}$ under pressure is a novel and interesting result. It is known that broadband emission in $2 \mathrm{D}$ perovskites is closely related to structural 
distortion induced by the organic spacers and by a wise chemical selection it is possible to modulate such an effect. ${ }^{30,31}$ In addition, we recently demonstrated that Ge-based 2D perovskite have an intrinsic higher degree of octahedra distortion in the bond-length distribution affecting the optical properties. $^{27}$ The emergence of broadband distortion around $2.1 \mathrm{GPa}$ is also most probably correlated to the emergence of the novel monoclinic phase with reduced $c$-axes (majority phase) which nicely couples to the trend of the band gap shown in Figure $7 \mathrm{~b}$. The first evidence of broadband emission by a $2 \mathrm{D}$ Ge-containing perovskite under moderate pressure is important to design suitable lead-free materials with analogous properties by playing with the chemical nature of the organic spacer at ambient pressure. Minimal previous data on pressure-induced broadband emission on 2D perovskite (all lead-based and containing bromide) have been reported to date, namely on $\mathrm{PEA}_{2} \mathrm{PbBr}_{4}, \mathrm{BA}_{2} \mathrm{PbBr}_{4}$, and $(4 \mathrm{BrPhMA})_{2} \mathrm{PbBr}_{4} .{ }^{32-34}$ These results, together with the chemical modulation on $2 \mathrm{D}$ perovskites leading to broadband emission, points towards the consensus about its origin by self-trapped excitions (STEs) generated by lattice structural distortions. ${ }^{35}$ The pressure-induced emergence of wide emission in $\mathrm{DA}_{2} \mathrm{GeI}_{4}$, after the transition from the orthorhombic to the monoclinic phase, strongly suggests that an increase of structural distortion plays a key role. The different behavior in the two perovskites investigated here, namely $\mathrm{DA}_{2} \mathrm{PbI}_{4}$ (narrow emission) and $\mathrm{DA}_{2} \mathrm{GeI}_{4}$ (broad emission) having a different central atom, but showing similar structural features at ambient and under applied pressure, is most likely correlated to the more significant distortion induced by germanium on octahedral bond elongation and bond angle variance, and less on the $\mathrm{Ge}-\mathrm{Br}-\mathrm{Ge}$ bond angle, with respect to $\mathrm{Pb}$-containing perovskite. ${ }^{27}$ This also couples to the dependance of the broadband emission with the increase of out-of-plane distortion in similar lead-based 2D perovskites. ${ }^{27}$ It is also known that native (or structural related) defects may contribute to self-trapping phenomena related to broad PL, and it is possible that this phenomenon may contribute to the observed high-pressure optical properties evolution of $\mathrm{DA}_{2} \mathrm{GeI}_{4}$, since in general lead-free materials are less defect tolerant. ${ }^{30}$ Further evidence about the presence of STEs in $\mathrm{DA}_{2} \mathrm{GeI}_{4}$, as the source of broadband emission, was corroborated by low-temperature PL 
measurements (Figure 8b). While still being a non-PL emitter at room temperature, a broad PL signal centered around $630 \mathrm{~nm}$ (with a quantum yield around 17\%) appears at $77 \mathrm{~K}$, a temperature where the photogenerated carriers can form stable self-trapped excitons within the distorted lattice. The broad emission at low temperature has a smaller Stokes shift with respect to the one induced by pressure, being peaked around $630 \mathrm{~nm}$, possibly due to the different extent of the lattice distortions induced by temperature and pressure. These data allow us to conclude that the presence of STEs is clearly a key characteristic of $\mathrm{DA}_{2} \mathrm{GeI}_{4}$ and that a broad emission can switched on by lowtemperature and high-pressure. As mentioned above, the peculiar octahedral distortion of Ge-based 2D perovskites seems to be the source of this effect. Further experimental and computational work will be carried out to get a solid microscopic description of the STEs nature in $\mathrm{DA}_{2} \mathrm{GeI}_{4}$ but it is clear that the present results open a new avenue to search broad - and possibly white - emitters in lead-free layered perovskites. 


\section{Conclusion}

We investigated the structural stability and optical properties of $\mathrm{DA}_{2} \mathrm{PbI}_{4}$ and $\mathrm{DA}_{2} \mathrm{GeI}_{4}$ with the aim to provide the evidence of pressure-induced phenomena in 2D perovskites characterized by a long alkyl chain and highlight the role of a different central atom. In this work we also reported a novel lead-free $2 \mathrm{D}$ perovskite, namely $\mathrm{DA}_{2} \mathrm{GeI}_{4}$, and we afforded the first high-pressure study of a layered Ge-containing phase.

From a structural point of view, both perovskites present, at ambient pressure, an orthorhombic crystal structure which converts to a monoclinic symmetry at relatively low pressure values $(<2 \mathrm{GPa})$. After the phase transition, further increase of pressure leads, for both materials, to the separation in two monoclinic phases, with one of the two having a distinct shorter $c$-axis. The present data, together with the few reports on pressure response of $2 \mathrm{D}$ perovskites, seems to indicate that phase separation might be a quite common behavior in these systems, differently from 3D perovskites, possibly due to the soft organic spacer which disorders and even melts by increasing pressure. The most remarkable difference between the two samples has been found in the evolution of the optical properties as a function of applied pressure. $\mathrm{DA}_{2} \mathrm{PbI}_{4}$ shows a progressive red shift of the band gap from $2.28 \mathrm{eV}$ at ambient conditions, to $1.64 \mathrm{eV}$ at $11.5 \mathrm{GPa}$ (maximum pressure studied). PL emission is narrow and clearly composed by two components, with the second one appearing in concomitance with the phase separation, significantly shifted to lower-energy. Maximum PL intensity if found around 3GPa. Both structural and optical response as a function of pressure are different with respect to $\mathrm{BA}_{2} \mathrm{PbI}_{4}$ (the other only alkyl-chain containing $2 \mathrm{D}$ perovskite studied to date) suggesting that a systematic study of the role of chain length on these lead-based 2D perovskites is worthy to elucidate the structure-property correlation as a function of pressure and number of carbon atoms of the spacer.

$\mathrm{DA}_{2} \mathrm{GeI}_{4}$ optical properties under pressure revealed a transition from a non-PL system at ambient pressure to a clear broadband emitter, with a large stoke shift, with an intensity maximum 
at about 3.7 GPa. Absorption measurements shows a first sudden blue-shift (at the orthorhombic to monoclinic phase transition) followed by a continuous red-shift. Wide emission with FWHM around $170 \mathrm{~nm}$ at about $730 \mathrm{~nm}$ in a 2D perovskite containing germanium is a novel result. While we already determined on other systems that there is an increased octahedral distortion induced by $\mathrm{Ge}$, such evidence of wide emission by a moderate pressure in a lead-free $2 \mathrm{D}$ perovskite is a result which deserves deep further investigation. By the appropriate choice of an organic spacer, capable of mimicking through chemical pressure a similar distortion degree, it will be possible to design efficient wide or even white lead-free emitters.

To conclude, our study provided novel insight into the role of alkyl chain length on the pressure-induced effects in lead-based 2D perovskites and unveiled a novel behavior on Ge-based systems, particularly in the emergence of broadband emission. It seems important to further extend the scope of the present study to both lead-based systems, to clarify the role of chain length on the structural and optical properties change with pressure, and, even more importantly, to analogous Ge-containing 2D perovskites to understand the extent and origin of broadband emission in order to be able to design suitable materials at ambient condition through chemical pressure effect. 


\section{Acknowledgements}

We thank Mr. Enrico Bassani (CNR-ICMATE) for help in collecting temperature resolved XRD patterns. We thank Elettra Synchrotron for providing beam-time. One of us (B.J.) acknowledge the "Xpress-PLUS" internal project from Elettra Sincrotrone Trieste. 


\section{References}

1. Postorino, P., and Malavasi, L. (2017). Pressure-Induced Effects in Organic-Inorganic Hybrid Perovskites. J. Phys. Chem. Lett. 8, 2613-2622.

2. Jaffe, A., Lin, Y., and Karunadasa, H.I. (2017). Halide Perovskites under Pressure: Accessing New Properties through Lattice Compression. ACS Energy Lett. 2, 1549-1555.

3. Li, Q., Zhang, L., Chen, Z., and Quan, Z. (2019). Metal halide perovskites under compression. J. Mater. Chem. A 7, 16089-16108.

4. Shi, Y., Zhou, Y., Ma, Z., Xiao, G., Wang, K., and Zou, B. (2020). Structural regulation and optical behavior of three-dimensional metal halide perovskites under pressure. J. Mater. Chem. C 8, 12755-12767.

5. Szafrański, M., and Katrusiak, A. (2017). Photovoltaic Hybrid Perovskites under Pressure. J. Phys. Chem. Lett. 8, 2496-2506.

6. Coduri, M., Shiell, T.B., Strobel, T.A., Mahata, A., Cova, F., Mosconi, E., De Angelis, F., and Malavasi, L. (2020). Origin of pressure-induced band gap tuning in tin halide perovskites. Mater. Adv. 1, 2840-2845.

7. Coduri, M., Strobel, T.A., Szafrański, M., Katrusiak, A., Mahata, A., Cova, F., Bonomi, S., Mosconi, E., De Angelis, F., and Malavasi, L. (2019). Band Gap Engineering in MASnBr3 and CsSnBr3 Perovskites: Mechanistic Insights through the Application of Pressure. J. Phys. Chem. Lett. 10, 7398-7405.

8. Seo, D.-K., Gupta, N., Whangbo, M.-H., Hillebrecht, H., and Thiele, G. (1998). PressureInduced Changes in the Structure and Band Gap of CsGeX3 $(\mathrm{X}=\mathrm{Cl}, \mathrm{Br})$ Studied by Electronic Band Structure Calculations. Inorg. Chem. 37, 407-410.

9. Morana, M., and Malavasi, L. (2021). Pressure Effects on Lead-Free Metal Halide Perovskites: a Route to Design Optimized Materials for Photovoltaics. Sol. RRL, 2100550.

10. Li, X., Hoffman, J.M., and Kanatzidis, M.G. (2021). The 2D Halide Perovskite Rulebook: How the Spacer Influences Everything from the Structure to Optoelectronic Device Efficiency. Chem. Rev. 121, 2230-2291.

11. Saidaminov, M.I., Mohammed, O.F., and Bakr, O.M. (2017). Low-Dimensional-Networked Metal Halide Perovskites: The Next Big Thing. ACS Energy Lett. 2, 889-896.

12. Zhou, C., Lin, H., He, Q., Xu, L., Worku, M., Chaaban, M., Lee, S., Shi, X., Du, M.-H., and Ma, B. (2019). Low dimensional metal halide perovskites and hybrids. Materials Science and Engineering: R: Reports 137, 38-65.

13. Han, Y., Yue, S., and Cui, B. (2021). Low-Dimensional Metal Halide Perovskite Crystal Materials: Structure Strategies and Luminescence Applications. Adv. Sci. 8, 2004805.

14. Gao, L., You, J., and Liu, S. (Frank) (2021). Superior photovoltaics/optoelectronics of twodimensional halide perovskites. Journal of Energy Chemistry 57, 69-82. 
15. Zhang, L., Liu, Y., Yang, Z., and Liu, S. (Frank) (2019). Two dimensional metal halide perovskites: Promising candidates for light-emitting diodes. Journal of Energy Chemistry 37, 97-110.

16. Wang, H.-P., Li, S., Liu, X., Shi, Z., Fang, X., and He, J.-H. (2021). Low-Dimensional Metal Halide Perovskite Photodetectors. Advanced Materials 33, 2003309.

17. Zhang, L., Wang, K., Lin, Y., and Zou, B. (2020). Pressure Effects on the Electronic and Optical Properties in Low-Dimensional Metal Halide Perovskites. J. Phys. Chem. Lett. 11, 4693-4701.

18. Matsuishi, K., Ishihara, T., Onari, S., Chang, Y.H., and Park, C.H. (2004). Optical properties and structural phase transitions of lead-halide based inorganic-organic 3D and 2D perovskite semiconductors under high pressure. phys. stat. sol. (b) 241, 3328-3333.

19. Yin, T., Liu, B., Yan, J., Fang, Y., Chen, M., Chong, W.K., Jiang, S., Kuo, J.-L., Fang, J., Liang, P., et al. (2019). Pressure-Engineered Structural and Optical Properties of TwoDimensional $\left(\mathrm{C}_{4} \mathrm{H}_{9} \mathrm{NH}_{3}\right)_{2} \mathrm{PbI}{ }_{4}$ Perovskite Exfoliated nm-Thin Flakes. J. Am. Chem. Soc. $141,1235-1241$.

20. Yuan, Y., Liu, X., Ma, X., Wang, X., Li, X., Xiao, J., Li, X., Zhang, H., and Wang, L. (2019). Large Band Gap Narrowing and Prolonged Carrier Lifetime of $\left(\mathrm{C}_{4} \mathrm{H}_{9} \mathrm{NH}_{3}\right)_{2} \mathrm{PbI}_{4}$ under High Pressure. Adv. Sci. 6, 1900240.

21. Matsuishi, K., Suzuki, T., Onari, S., Gregoryanz, E., Hemley, R.J., and Mao, H.K. (2001). Excitonic States of Alkylammonium Lead-Iodide Layered Perovskite Semiconductors under Hydrostatic Pressure to 25 GPa. phys. stat. sol. (b) 223, 177-182.

22. Sichert, J.A., Hemmerling, A., Cardenas-Daw, C., Urban, A.S., and Feldmann, J. (2019). Tuning the optical bandgap in layered hybrid perovskites through variation of alkyl chain length. APL Materials 7, 041116.

23. Billing, D.G., and Lemmerer, A. (2007). Synthesis, characterization and phase transitions in the inorganic-organic layered perovskite-type hybrids $\left[\left(\mathrm{C}_{n} \mathrm{H}_{2 n+1} \mathrm{NH}_{3}\right)_{2} \mathrm{PbI} 4\right], n=4,5$ and 6. Acta Crystallogr B Struct Sci 63, 735-747.

24. Billing, D.G., and Lemmerer, A. (2008). Synthesis, characterization and phase transitions of the inorganic-organic layered perovskite-type hybrids $[(\mathrm{CnH} 2 \mathrm{n}+1 \mathrm{NH} 3) 2 \mathrm{PbI} 4](\mathrm{n}=12,14,16$ and 18). New J. Chem. 32, 1736.

25. Lemmerer, A., and Billing, D.G. (2012). Synthesis, characterization and phase transitions of the inorganic-organic layered perovskite-type hybrids $\left[\left(\mathrm{C}_{\mathrm{n}} \mathrm{H}_{2 \mathrm{n}+1} \mathrm{NH}_{3}\right)_{2} \mathrm{PbI} 4\right], n=7,8,9$ and 10. Dalton Trans. 41, 1146-1157.

26. Malavasi, L., Morana, M., and Chiara, R. (2021). Germanium-based Halide Perovskites: Materials, Properties, and Applications. ChemPlusChem, cplu.202100191.

27. Malavasi, L., Bindi, L., Coduri, M., De Angelis, F., Fracassi, F., Gaboardi, M., Listorti, A., Mahata, A., Milanese, C., Morana, M., et al. (2021). Role of Spacer Cations and Structural Distortion in Two-Dimensional Germanium Halide Perovskites. J. Mater. Chem. C. 
28. Liu, G., Gong, J., Kong, L., Schaller, R.D., Hu, Q., Liu, Z., Yan, S., Yang, W., Stoumpos, C.C., Kanatzidis, M.G., et al. (2018). Isothermal pressure-derived metastable states in 2D hybrid perovskites showing enduring bandgap narrowing. Proc Natl Acad Sci USA 115, 8076-8081.

29. Xu, J., Maxwell, A., Wei, M., Wang, Z., Chen, B., Zhu, T., and Sargent, E.H. (2021). Defect Tolerance of Mixed B-Site Organic-Inorganic Halide Perovskites. ACS Energy Lett., 42204227.

30. Cortecchia, D., Neutzner, S., Srimath Kandada, A.R., Mosconi, E., Meggiolaro, D., De Angelis, F., Soci, C., and Petrozza, A. (2017). Broadband Emission in Two-Dimensional Hybrid Perovskites: The Role of Structural Deformation. J. Am. Chem. Soc. 139, 39-42.

31. Smith, M.D., Jaffe, A., Dohner, E.R., Lindenberg, A.M., and Karunadasa, H.I. (2017). Structural origins of broadband emission from layered $\mathrm{Pb}-\mathrm{Br}$ hybrid perovskites. Chem. Sci. 8 , 4497-4504.

32. Shen, P., Vogt, T., and Lee, Y. (2020). Pressure-Induced Enhancement of Broad-Band White Light Emission in Butylammonium Lead Bromide. J. Phys. Chem. Lett. 11, 4131-4137.

33. Zhang, L., Wu, L., Wang, K., and Zou, B. (2019). Pressure-Induced Broadband Emission of 2D Organic-Inorganic Hybrid Perovskite $\left(\mathrm{C}_{6} \mathrm{H}_{5} \mathrm{C}_{2} \mathrm{H}_{4} \mathrm{NH}_{3}\right)_{2} \mathrm{PbBr}_{4}$. Adv. Sci. 6, 1801628.

34. Gómez, V., Klyatskaya, S., Fuhr, O., Kalytchuk, S., Zbořil, R., Kappes, M., Lebedkin, S., and Ruben, M. (2020). Pressure-Modulated Broadband Emission in 2D Layered Hybrid PerovskiteLike Bromoplumbate. Inorg. Chem. 59, 12431-12436.

35. Smith, M.D., and Karunadasa, H.I. (2018). White-Light Emission from Layered Halide Perovskites. Acc. Chem. Res. 51, 619-627. 